Pacific Journal of Mathematics

PRESERVATION OF LOCAL PROPERTIES AND CHAIN
CONDITIONS IN COMMUTATIVE GROUP RINGS

DAVID C. LAN 


\title{
PRESERVATION OF LOCAL PROPERTIES AND CHAIN CONDITIONS IN COMMUTATIVE GROUP RINGS
}

\author{
DAVID C. LANTZ
}

This note gives necessary and sufficient conditions for a commutative group ring to be locally Noetherian, locally Cohen-Macaulay, locally Gorenstein, or locally regular, and sufficient conditions for it to satisfy the chain condition or the second chain condition for prime ideals.

The purpose of this note is to extend certain results from [1] (and, as there, all rings are commutative with identity). The object in that paper was the description of a certain UFD, but the authors' results included necessary and sufficient conditions for $S[G]$ to be locally Noetherian for $G$ an abelian group of finite torsion-free rank and $S$ either a field or the ring of integers. They also gave conditions for a localization of $S[G]$ to be Cohen-Macaulay or regular for such coefficient rings $S$. Their proof is quite general; we use it here to give necessary and sufficient conditions for $S[G]$ to be locally Noetherian, locally Cohen-Macaulay, locally Gorenstein, or locally regular, for a general coefficient ring. It was also shown in [1] that $S[G]$ satisfies the second chain condition when $S$ is a field and $G$ is an abelian group of finite torsion-free rank; and Brewer suggested that this result might also extend to more general coefficient rings. We prove the result here for $S$ a Noetherian Hilbert domain satisfying the second chain condition, and we see by example that the hypotheses "Noetherian" and "Hilbert" are both needed.

Our first lemma is Lemma 2 in [1].

LEMMA 1. If $G$ is an abelian group of finite torsion-free rank and $F$ is a free subgroup of $G$ such that $G / F$ is torsion and $(G / F)_{p}$ is finite for some prime $p$, then $G_{p}$ is finite and $G=G_{p} \oplus H$ where $H$ contains a free subgroup $F_{1}$ such that $H / F_{1}$ is torsion and $\left(H / F_{1}\right)_{p}=0$.

We also need two facts about the Cohen-Macaulay property in commutative group rings.

LEMma 2. Let $S$ be a Cohen-Macaulay ring, $G$ an abelian group of finite torsion-free rank, and $M$ a prime ideal in $S[G]$. If $S[G]_{M}$ is Noetherian, then $S[G]_{M}$ is Cohen-Macaulay.

Proof. Let $F$ be a free subgroup of $G$ such that $G / F$ is torsion, 
and set $M_{0}=M \cap S[F]$. Since $S[G]$ is integral over $S[F]$, rank $M \leqq$ rank $M_{0}$. Since $S[G]_{M}$ is a faithfully flat extension of $S[F]_{M_{0}}$, a regular $S[F]_{M_{0}}$-sequence is a regular $S[G]_{M}$-sequence, so grade $M S[G]_{M} \geqq$ grade $M_{0} S[F]_{M_{0}}$. Now $S[F]_{M_{0}}$ is a localization of $S\left[X_{1}, \cdots, X_{t}\right]$ where $t=\operatorname{rank} F$, so it is Cohen-Macaulay. Thus

$$
\begin{aligned}
\operatorname{rank} M S[G]_{M} & \geqq \operatorname{grade} M S[G]_{M} \geqq \operatorname{grade} M_{0} S[F]_{M 0} \\
& =\operatorname{rank} M_{0} S[F]_{M_{0}}=\operatorname{rank} M_{0} \geqq \operatorname{rank} M=\operatorname{rank} M S[G]_{M} .
\end{aligned}
$$

Lemma 3. Let $(S, P)$ be a Cohen-Macaulay ring of type $r$ (in the sense of [3], Definition 1.20), and let $G$ be a finitely generated abelian group. Let $M$ be a prime ideal of $S[G]$ lying over $P$. Then $S[G]_{M}$ is also Cohen-Macaulay of type $r$.

Proof. Since $G$ is finitely generated, $S[G]$ is Noetherian, so $S[G]_{M}$ is Noetherian, and hence Cohen-Macaulay by Lemma 2. Let $F$ be a free group and $F \rightarrow G$ a group epimorphism. Let $g_{1}, \cdots, g_{r}$ be a basis for the kernel of $F \rightarrow G$, and denote by $M^{\prime}$ the inverse image of $M$ under the induced epimorphism $S[F] \rightarrow S[G]$. Now

$$
S[F]_{M^{\prime}} / P S[F]_{M^{\prime}}=(S / P)[F]_{\overline{M^{\prime}}},
$$

a localization of a polynomial ring over a field, and hence of type 1 . By [3], Satz 1.24, $S[F]_{M}$, has type $r$. Now $X^{g_{i+1}}-1$ is regular in $S[F] /\left(X^{g_{1}}-1, \cdots, X^{g_{i}}-1\right)=S\left[F /\left(g_{1}, \cdots, g_{2}\right)\right]$, and each $X^{g_{i}}-1$ is in $M^{\prime}$, so $X^{g_{1}}-1, \cdots, X^{g_{r}}-1$ is a regular $S[F]_{M^{\prime}}$-sequence. By [3], 1.22, $S[G]_{M}=S\left[F /\left(g_{1}, \cdots, g_{r}\right)\right]_{M}=S[F]_{M^{\prime}} /\left(X^{g_{1}}-1, \cdots, X^{g_{r}}-1\right)_{M^{\prime}}$ also has type $r$.

Let $S$ be a commutative ring, $G$ an abelian group of finite torsionfree rank, and $\widetilde{M}$ a prime ideal of $S[G]$. Write $P=\widetilde{M} \cap S$ and pick a free subgroup $F$ of $G$ such that $G / F$ is torsion. The ideal $\widetilde{M}$ contains at most one prime integer $p$, as represented in the prime subring of $S$. (If $\widetilde{M}$ contains no prime integer, the last part of each statement below is vacuous.)

Proposition 1. The ring $S[G]_{\tilde{M}}$ is:

(1) Noetherian if $S_{p}$ is Noetherian and $(G / F)_{p}$ is finite.

(2) Cohen-Macaulay of type $r$ if $S_{P}$ is Cohen-Macaulay of type $r$ and $(G / F)_{p}$ is finite.

(3) regular is $S_{P}$ if regular, $(G / F)_{p}$ is finite, and $G_{p}=0$.

Proof. (1) If $p \in \tilde{M}$, write $G=G_{p} \oplus H$ as in Lemma 1, and assume $F \subseteq H$ and $(H / F)_{p}=0$; otherwise let $H=G$. Write $R=S[H]$ and $R_{0}=S[F]$, and set $M=\widetilde{M} \cap R$ and $M_{0}=\widetilde{M} \cap R_{0}$. We show first 
that $M R_{M}=M_{0} R_{M}:$ For $m \in M, m \in T=S[E]$ for some finitely generated subgroup $E$ of $H$ containing $F$. Since $E / F$ is finite, we can write $R_{0} \subseteq R_{1} \subseteq \cdots \subseteq R_{s}=T$ where $R_{j}=S\left[E_{j}\right]$ and $E_{j}$ is generated by $E_{j-1}$ and $h_{j} \in E\left(E_{0}=F\right)$. Set $M_{j}=M \cap R_{j}$. The order $k$ of $h_{j}$ in $E / F$ is prime to $p$, so $k\left(X^{h_{j}}\right)^{k-1}$ is a unit in $\left(R_{j}\right)_{M_{j}}$; and $\left(X^{h_{j}}\right)^{k} \in R_{j_{-1}}$, so by [5], Theorem (38.6), $M_{j-1}\left(R_{j}\right)_{M_{j}}=M_{j}\left(R_{j}\right)_{M_{j}}$. Thus $m / 1 \in M_{0}\left(R_{s}\right)_{M_{s}}=$ $M_{0} T_{M \cap T} \subseteq M_{0} R_{M}$.

Let $I$ be a finitely generated ideal of $R$ and $r / 1 \in \bigcap_{q=0}^{\infty}\left(I R_{M}+\right.$ $M^{q} R_{M}$ ). Pick a new finitely generated subgroup $E$ of $H$ containing $F$ such that $r$ and a set of generators for $I$ lie in $S[E]=T$. Since $T_{M \cap T}$ is Noetherian, and $R_{M}$ is faithfully flat over it, and $R_{0} \leqq T \leqq R$, we have $r / 1 \in \bigcap_{q=0}^{\infty}\left[(I \cap T)+(M \cap T)^{q}\right] R_{M} \cap T_{M \cap T}=\bigcap_{q=0}^{\infty}[(I \cap T)+$ $\left.(M \cap T)^{q}\right] T_{M \cup T}=(I \cap T) T_{M \cap T} \cong(I \cap T) R_{M}=I R_{M}$. With $I=0$, this shows $\bigcap_{q=0}^{\infty} M^{q} R_{M}=0$, so that $R_{M}$ is a "local ring which may not be Noetherian", and for arbitrary $I$ it shows that every finitely generated ideal of $R_{M}$ is closed in the $M R_{M}$-adic topology. By [5], Theorem (31.8), $R_{M}$ is Noetherian. Now $S[G]$ is a finitely generated $R$-module, so $(R \backslash M)^{-1} S[G]$ is a finitely generated $R_{M}$-module and hence is Noetherian; so $S[G]_{\tilde{M}}$ is Noetherian.

(2) By Lemma 2, $S[G]_{\tilde{M}}$ is Cohen-Macaulay. Now in the notation of (1), $\left(R_{0}\right)_{M_{0}}$ has type $r$ by Lemma 3. Since $M_{0} R_{M}=M R_{M}$, Satz 1.24 of [3], applied to $\left(R_{0}\right)_{M_{0}} \subseteq R_{M}$ yields the fact that $R_{M}$ has type $r$. If $G=H$, we are finished. If $G=G_{p} \oplus H$, we can apply Lemma 3 to $S[G]_{\tilde{M}}$ as a localization of $R_{M}\left[G_{p}\right]$.

(3) In the notation of (1), $R=S[G]$ and $M=\tilde{M}$. Now $\left(R_{0}\right)_{M_{0}}$ is a localization of $S_{P}\left[X_{1}, \cdots, X_{t}\right]$ where $t=\operatorname{rank} F$, and so is regular; and $M_{0} R_{M}=M R_{M}$ (together with the fact that $R_{M}$ is a faithfully flat extension of $\left.\left(R_{0}\right)_{M_{0}}\right)$ shows that $R_{M}$ is regular.

The proof of (1) in Proposition 1 (and of (1) in the theorem below) is the proof of Theorem $\mathrm{A}$ in [1]. We repeat it here to establish notation for the other parts, and to make the minor changes needed to prove this proposition. Lady has pointed out the similarity of this argument to that used in the proof of the lemma in the middle of page 95 in [8].

Letting $\tilde{M}$ vary over the maximal ideals of $S[G]$ yields one direction of the theorem below. For the reversal of one implication we shall need:

Lemma 4. Let $S$ be a ring and $G$ be an abelian group. Let $P$ be a prime ideal of $S$ and $H$ be a finite subgroup of $G$. Denote by $M$ the (prime) ideal of $S[G]$ generated by $P$ and the elements $X^{g}-1$, $g \in G$. Then $\sum_{g \in H} X^{g}$ is nonzero in $S[G]_{M}$. 
Proof. For any element $f=\sum_{g \in G} a_{g} X^{g}$ of $S[G],\left(\sum_{g \in H} X^{g}\right) f=$ $\sum_{g \in G}\left(\sum_{g^{\prime} \in g+H} a_{g^{\prime}}\right) X^{g}$, which is 0 only if $\sum_{g^{\prime} \in g+H} a_{g^{\prime}}=0$ for each coset $g+H$ of $H$ in $G$. This condition implies that $\sum_{g \in G} a_{g}=0$, an element of $P$, so $f \in M$. It follows that ann $\left(\sum_{g_{\in} H} X^{g}\right) \subseteq M$.

THEOREM. Let $S$ be a commutative ring with identity and $\Omega$ be the set of prime integers which are nonunits in $S$. Let $G$ be an abelian group of finite torsion-free rank and $F$ a free subgroup of $G$ such that $G / F$ is torsion. Then $S[G]$ is:

(1) Locally Noetherian if and only if $S$ is locally Noetherian and, for each $p$ in $\Omega,(G / F)_{p}$ is finite.

(2) Locally Cohen-Macaulay if and only if $S$ is locally CohenMacaulay and for each $p$ in $\Omega,(G / F)_{p}$ is finite.

(3) Locally Gorenstein if and only if $S$ is locally Gorenstein and, for each $p$ in $\Omega,(G / F)_{p}$ is finite.

(4) Locally regular if and only if $S$ locally regular and, for each $p$ in $\Omega,(G / F)_{p}$ is finite and $G_{p}=0$.

Moreover, if $S$ is locally Cohen-Macaulay and $P$ is a prime ideal in $S[G]$ for which $S[G]_{P}$ is Noetherian, then $S[G]_{P}$ is CohenMacaulay.

Proof. Sufficiency of the conditions follows from Proposition 1 (including (3), since a Gorenstein ring is a Cohen-Macaulay ring of type 1). For the necessity, let $P$ be a maximal ideal of $S$, and denote by $M$ the maximal ideal of $S[G]$ generated by $P$ and all elements of the form $X^{g}-1, g \in G$. Then $S[G]_{M}$ is a faithfully flat extension of $S_{P}$, so if $S[G]_{M}$ is Noetherian, or Cohen-Macaulay, or Gorenstein, or regular, then $S_{P}$ has the same property. Thus we need only verify the assertions about the group.

If $(G / F)_{p}$ is infinite for some $p$ in $\Omega$, then let $P$ be a maximal ideal of $S$ containing $p$ and form $M$ as in the last paragraph. We show $M S[G]_{M}$ is not finitely generated: Let $I$ be any finitely generated ideal contained in $M$, and let $H$ be a finitely generated subgroup of $G$ containing $F$ for which a set of generators for $I$ is contained in $S[H]$. Since $H$ is finitely generated, $G / H$ contains an element $g+H$ of order $p$. Since $I \subseteq M$, the image of $I$ in $S[G] /\left(\left\{X^{g}-1: g \in H\right\}\right)=$ $S[G / H]$ is 0 , but since ann $\left(X^{g+H}-1\right)=\left(\sum_{n=0}^{p-1} X^{n g+H}\right)$ is contained in the image of $M$, we have that the image of any $f$ in $S[G] \backslash M$ does not annihilate $X^{g+H}-1$, so $f\left(X^{g}-1\right) \notin I$, so $X^{g}-1 \notin I S[G]_{M}$. Thus $I S[G]_{M} \neq M S[G]_{M}$.

Assume $G_{p} \neq 0$ for some $p$ in $\Omega$, and pick a maximal ideal $P$ of $S$ containing $p$; form $M$ as above. Pick an element $g$ of $G_{p}$ of order $p$ and consider $\left(X^{g}-1\right)\left(\sum_{n=0}^{p-1} X^{n g}\right)=0$. Neither factor is 0 , even in 
$S[G]_{M}$, so $S[G]_{M}$ is not a domain, and thus cannot be regular.

It may be possible to prove a version of the theorem's "Moreover" assertion with "Gorenstein" replacing "Cohen-Macaulay" but another proof will be required, since the condition of faithful flatness seems not to transfer enough properties to the larger ring.

We turn now to chain conditions on commutative group rings. The key facts used are drawn from the work of Ratliffe.

Lemma 5. Let $R$ be a ring which satisfies the second chain condition and whose total quotient ring is 0-dimensional, and let $S$ be an integral ring extension which is torsion-free as an $R$-module. Then $S$ also satisfies the second chain condition.

Proof. By [7], Corollary 2.17, this will follow if we can show that every minimal prime ideal in $S$ meets $R$ in a minimal prime; but if $P$ is a minimal prime in $S$ and $P \cap R$ is not minimal in $R$, then $\{t x: t$ is a regular element of $R, x \in S \backslash P\}$ is a multiplicatively closed set in $S$ missing 0 but properly containing $S \backslash P$, a contradiction.

Lemma 6. Let $R$ be a Hilbert ring and $x \in R$, not nilpotent. Then:

(1) $R_{x}$, the ring of quotients of $R$ with denominators powers of $x$, is Hilbert; and

(2) the maximal ideals of $R_{x}$ are the extensions of maximal ideals of $R$ which do not contain $x$.

Proof. A prime in $R_{x}$ has the form $P R_{x}$ where $P$ is prime in $R$ and $x \notin P$. Now $P$ is the intersection of maximal ideals $M_{z}$ of $R$; it suffices to show $P R_{x}=\bigcap_{\lambda}\left(M_{\lambda}\right) R_{x}$, and one inclusion is clear. Let $a / x^{n} \in \bigcap_{\lambda}\left(M_{\lambda}\right) R_{x}$; then for each $\lambda$ there is a positive integer $k_{\lambda}$ for which $x^{k} \alpha \in M_{\lambda}$. If $x \notin M_{\lambda}$, then $a \in M_{\lambda}$ so $x a$ is in each $M_{\lambda}$. Thus $x a \in P$, so $a / x^{n}=x a / x^{n+1} \in P R_{x}$. For (2), suppose $P R_{x}$ is maximal. The prime $P$ in $R$ is an intersection of maximals, one of which misses $x$ and hence survives in $R_{x}$, so $P$ is maximal.

Proposition 2. Let $R$ be a Noetherian domain and $G$ an abelian group of finite torsion-free rank. If $R$ satisfies the chain condition, then so does $R[G]$. If $R$ is also Hilbert and satisfies the second chain condition, then $R[G]$ satisfies the second chain condition.

Proof. Let $F$ be a free subgroup of $G$ such that $G / F$ is torsion, and set $t=\operatorname{rank} F$. If $R$ is a Noetherian domain satisfying the chain condition, then by [6], Theorem 3.6, $R\left[X_{1}, \cdots, X_{t}\right]$ satisfies the chain 
condition. By [5], Theorem (34.1), the chain condition passes to the localization $R\left[X_{1}, \cdots, X_{t}\right]_{X_{1} \cdots X_{t}}=R[F]$ and then to the integral extension $R[G]$.

As noted in [5], page 123, in a domain of finite altitude the second chain condition is equivalent to the chain condition and equidimensionality. If $R$ is a Noetherian Hilbert domain satisfying the second chain condition, then each stage in

$$
R \subseteq R\left[X_{1}\right] \subseteq \cdots \leqq R\left[X_{1}, \cdots, X_{t}\right] \subseteq R[F] \subseteq R[G]
$$

satisfies the chain condition, so it suffices to show that all but $R[G]$ are equidimensional. For the polynomial extensions, let $M$ be a maximal ideal in $R[X]$; then $M \cap R$ is maximal in $R$ and $(M \cap R)[X] \neq$ $M, \quad$ so $\quad$ alt $R[X] \geqq \operatorname{rank} M \geqq \operatorname{rank}(M \cap R)[X]+1 \geqq \operatorname{rank}(M \cap R)+$ $1=$ alt $R+1=$ alt $R[X]$. Lemma 6 shows that $R[F]$ is equidimensional, and Lemma 5 yields the result.

To see that the hypotheses of "Noetherian" and "Hilbert" are needed, we give examples of one-dimensional domains $R$ for which equidimensionality fails in $R[G]$, even when $G$ is infinite cyclic:

(1) A discrete (rank one) valuation domain $V$ is Noetherian but not Hilbert, so $V[X]$ contains a maximal ideal lying over 0 in $V$. This ideal must have rank one, since only two primes in a chain in $V[X]$ can lie over the same prime in $V$; and it cannot contain $X$, so it survives in $V\left[X, X^{-1}\right]$. On the other hand, if $P$ is the maximal ideal in $V$, then $P[X]+(X-1)$ is a rank 2 maximal which survives in $V\left[X, X^{-1}\right]$.

(2) Let $D$ be the Hilbert domain of Example 1 in [2], and let $P$ and $Q$ be the ideals described. The maximal ideals $P[Z]+(Z-1)$ and $Q[Z]+(Z-1)$ of $D[Z]$ both survive in $D\left[Z, Z^{-1}\right]$, but one has rank 3 and the other rank 2.

ACKNOWLEDGMENT. The author wishes to thank his dissertation advisor, Professor James Brewer, for his help and encouragement, and Professor E. Lee Lady and the referee for their improvements.

\section{REFERENCES}

1. J. W. Brewer, D. L. Costa and E. L. Lady, Prime ideals and localization in commutative group rings, J. Algebra, 34 (1975), 300-308.

2. J. W. Brewer, P. R. Montgomery, E. A, Rutter and W. J. Heinzer, Krull dimension in polynomial rings, Conference on Commutative Algebra, Lawrence, Kansas, 1972. Lecture Notes in Mathematics \#311, New York: Springer-Verlag, 1972.

3. J. Herzog and E. Kunz, Der kanonische Modul eines Cohen-Macaulay-Rings, Lecture Notes in Mathematics \#238, New York: Springer-Verlag, 1971.

4. I. Kaplansky, Commutative Rings, Boston: Allyn and Bacon, 1970.

5. M. Nagata, Local Rings, New York: Interscience, 1962. 
6. L. Ratliffe, On quasi-unmixed local domains, the altitude formula, and the chain condition for prime ideals (I), Amer. J. Math., 91 (1969), 508-528.

7. - On quasi-unmixed local domains, the altitude formula, and the chain condition for prime ideals (II), Amer. J. Math., 92 (1970) 99-144.

8. M. Raynaud, Anneaux Locaux Henséliens, Lecture Notes in Mathematics \#169, New York: Springer-Verlag, 1970.

Received July 23, 1975 and in revised form November 11, 1975.

UNIVERSITY of Nebraska at OMAHA 



\section{PACIFIC JOURNAL OF MATHEMATICS}

EDITORS

RICHARD ARENS (Managing Editor)

University of California

Los Angeles, California 90024

R. A. BEAUMONT

University of Washington

Seattle, Washington 98105
J. DugundJI

Department of Mathematics

University of Southern California

Los Angeles, California 90007

D. Gilbarg and J. Milgram

Stanford University

Stanford, California 94305

\section{ASSOCIATE EDITORS}

E. F. BECKENBACH

B. H. NeumanN

F. WOLF

K. YosHIDA

\section{SUPPORTING INSTITUTIONS}

UNIVERSITY OF BRITISH COLUMBIA

UNIVERSITY OF SOUTHERN CALIFORNIA

CALIFORNIA INSTITUTE OF TECHNOLOGY

UNIVERSITY OF CALIFORNIA

STANFORD UNIVERSITY

UNIVERSITY OF TOKYO

MONTANA STATE UNIVERSITY

UNIVERSITY OF UTAH

UNIVERSITY OF NEVADA

WASHINGTON STATE UNIVERSITY

NEW MEXICO STATE UNIVERSITY

UNIVERSITY OF WASHINGTON

OREGON STATE UNIVERSITY

UNIVERSITY OF OREGON

OSAKA UNIVERSITY

AMERICAN MATHEMATICAL SOCIETY
NAVAL WEAPONS CENTER

Printed in Japan by International Academic Printing Co., Ltd., Tokyo, Japan 


\section{Pacific Journal of Mathematics}

\section{Vol. 63, No. $1 \quad$ March, 1976}

Ralph Artino, Gevrey classes and hypoelliptic boundary value problems ....... 1

B. Aupetit, Caractérisation spectrale des algèbres de Banach commutatives .... 23

Leon Bernstein, Fundamental units and cycles in the period of real quadratic

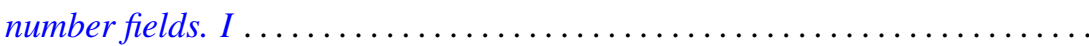

Leon Bernstein, Fundamental units and cycles in the period of real quadratic number fields. II.................................... 63

Robert F. Brown, Fixed points of automorphisms of compact Lie groups ........

Thomas Ashland Chapman, Concordances of noncompact Hilbert cube

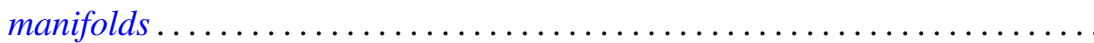

William C. Connett, V and Alan Schwartz, Weak type multipliers for Hankel

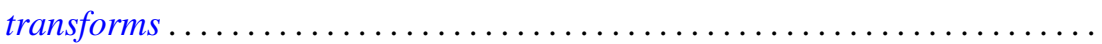

John Wayne Davenport, Multipliers on a Banach algebra with a bounded approximate identity .....................................

Gustave Adam Efroymson, Substitution in Nash functions ................ 137

John Sollion Hsia, Representations by spinor genera ..................

William George Kitto and Daniel Eliot Wulbert, Korovkin approximations in

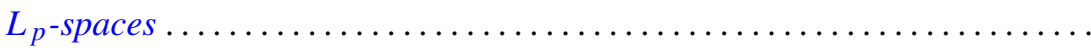

Eric P. Kronstadt, Interpolating sequences for functions satisfying a Lipschitz. condition ...........................................

Gary Douglas Jones and Samuel Murray Rankin, III, Oscillation properties of certain self-adjoint differential equations of the fourth order...

Takaŝi Kusano and Hiroshi Onose, Nonoscillation theorems for differential

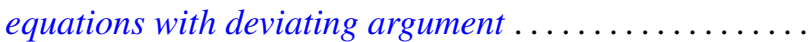

David C. Lantz, Preservation of local properties and chain conditions in commutative group rings. ...

Charles W. Neville, Banach spaces with a restricted Hahn-Banach extension property....

Norman Oler, Spaces of discrete subsets of a locally compact group ...

Robert Olin, Functional relationships between a subnormal operator and its minimal normal extension.

Thomas Thornton Read, Bounds and quantitative comparison theorems for nonoscillatory second order differential equations ...... .

Robert Horace Redfield, Archimedean and basic elements in completely distributive lattice-ordered groups...

Jeffery William Sanders, Weighted Sidon sets

Aaron R. Todd, Continuous linear images of pseudo-complete linear topological spaces.

J. Jerry Uhl, Jr., Norm attaining operators on $L^{1}[0,1]$ and the Radon-Nikodým property.

William Jennings Wickless, Abelian groups in which every endomorphism is a left multiplication. 